\title{
Some results on approximate solutions of variational inequality problems for inverse strongly monotone operators
}

\section{Shigeru lemoto*}

"Correspondence:

iemoto@tamacc.chuo-u.ac.jp Chuo University Suginami High

School, 2-7-1 Imagawa

Suginami-ku, Tokyo, 167-0035, Japan

\begin{abstract}
We present results on approximate solutions to variational inequality problems for an injective inverse strongly monotone operator. Our results are based on Edelstein's theorem (Edelstein in J. Lond. Math. Soc. 37:74-79, 1962).

MSC: 49J40; 47H10;47H09; 49K27

Keywords: variational inequality problem; fixed point; projected gradient method; hybrid steepest descent method; inverse strongly monotone operator; cocoercive operator; strongly monotone operator
\end{abstract}

\section{Introduction}

In this paper, we consider iterative algorithms for solving the variational inequality problem for a monotone operator $A$ over a nonempty closed convex subset $C$ of a real Hilbert space $H$ with the inner product $\langle\cdot, \cdot\rangle$ and its induced norm $\|\cdot\|$,

$$
\text { find } z \in C \text { such that }\langle y-z, A z\rangle \geq 0 \text { for all } y \in C \text {. }
$$

We denote the set of solutions of Problem (1) by $\operatorname{VI}(C, A)$. This sort of problem was first studied by Lions and Stampacchia [1] and is central to the study of nonlinear analysis. Problem (1) can be solved by using convex optimization techniques. A typical iterative procedure for doing so is the projected gradient method (PGM) [2,3], expressed as follows.

Theorem 1.1 ([2]) Let $C$ be a closed convex subset of a Hilbert space $H$ and $A$ be an LLipschitz continuous and $\beta$-strongly monotone operator sending $C$ into H. Furthermore, let $\left\{x_{n}\right\}$ be a sequence in $C$ defined by $x_{1} \in C$ and

$$
x_{n+1}=P_{C}(I-a A) x_{n}
$$

for $n=1,2, \ldots$, where $P_{C}$ is the metric projection onto $C, I$ is the identity mapping on $H$, and $a \in\left(0,2 \beta / L^{2}\right)$. Then $\left\{x_{n}\right\}$ converges strongly to a unique $v \in V I(C, A)$.

As (PGM) requires repetitive use of $P_{C}$, it works only when the explicit form of $P_{C}$ is known (e.g., $C$ is a closed ball or a closed cone). The following method, called the hybrid

(C) 2015 lemoto. This article is distributed under the terms of the Creative Commons Attribution 4.0 International License (http://creativecommons.org/licenses/by/4.0/), which permits unrestricted use, distribution, and reproduction in any medium, provided you give appropriate credit to the original author(s) and the source, provide a link to the Creative Commons license, and indicate if changes were made. 
steepest descent method (HSDM) [4], enables us to consider the case in which $C$ has a more complicated form.

Theorem 1.2 ([4]) Let $H$ be a Hilbert space and $S$ be a nonexpansive mapping on $H$ with $\operatorname{Fix}(S):=\{x \in H: S x=x\} \neq \emptyset$. Furthermore, let $A$ be an L-Lipschitz continuous and $\beta$ strongly monotone operator on $H$. In addition, let $a \in\left(0,2 \beta / L^{2}\right)$ and $\left\{c_{n}\right\}$ be a sequence in $(0,1]$ with $\lim _{n \rightarrow \infty} c_{n}=0$, and let $\left\{u_{n}\right\}$ be a sequence in $H$ defined by $u_{1} \in H$ and

$$
u_{n}=\left(I-c_{n} a A\right) S u_{n}
$$

for $n=1,2, \ldots$. Then $\left\{u_{n}\right\}$ converges strongly to a unique $v \in V I(\operatorname{Fix}(S), A)$.

Theorem 1.3 (HSDM [4]) Let H be a Hilbert space and S be a nonexpansive mapping on $H$ with $\operatorname{Fix}(S) \neq \emptyset$. Furthermore, let $A$ be an L-Lipschitz continuous and $\beta$-strongly monotone operator on $H, a \in\left(0,2 \beta / L^{2}\right)$, and $\left\{c_{n}\right\}$ be a sequence in $(0,1]$ that satisfies
(i) $\lim _{n \rightarrow \infty} c_{n}=0$,
(ii) $\sum_{n=1}^{\infty} c_{n}=\infty$,
(iii) $\lim _{n \rightarrow \infty} \frac{c_{n}-c_{n+1}}{c_{n+1}^{2}}=0$.

Let $\left\{x_{n}\right\}$ be a sequence in $H$ defined by $x_{1} \in H$ and

$$
x_{n+1}=\left(I-c_{n} a A\right) S x_{n}
$$

for $n=1,2, \ldots$. Then $\left\{x_{n}\right\}$ converges strongly to a unique $v \in V I(\operatorname{Fix}(S), A)$.

In 2003, Xu and Kim [5] replaced condition (iii) in Theorem 1.3 with $\lim _{n \rightarrow \infty}\left(c_{n} /\right.$ $\left.c_{n+1}\right)=1$, which includes the case of $c_{n}=1 / n$. The proofs of Theorems 1.2, 1.3 are based on the Banach contraction mapping principle [6]. In the Hilbert space setting, the Banach contraction mapping principle is as follows.

Theorem 1.4 ([6]) Let $C$ be a closed subset of a Hilbert space $H$ and $S$ be a strictly contractive mapping of $C$ into $H$; that is, there exists $r \in[0,1)$ such that

$$
\|S x-S y\| \leq r\|x-y\|
$$

for any $x, y \in C$. Let $\left\{x_{n}\right\}$ be a sequence in $C$ defined by $x_{1} \in C$ and $x_{n+1}=S x_{n}$ for $n=1,2, \ldots$. Then $\left\{x_{n}\right\}$ converges strongly to a unique fixed point $v \in C$ of $S$.

Motivated by the above theorems, we present some results on approximate solutions of variational inequality problems for an injective inverse strongly monotone operator. Our approach is as follows: Theorems 1.1-1.3 deal with the case in which $A$ is $L$-Lipschitz continuous and $\beta$-strongly monotone; that is, there exist $L \in(1, \infty)$ and $\beta \in(0,1)$ such that

$$
\|A x-A y\| \leq L\|x-y\| \quad \text { and } \quad \beta\|x-y\|^{2} \leq\langle x-y, A x-A y\rangle
$$


for all $x, y$ in the domain of $A$. Accordingly, we know that $A$ is injective and $\beta / L^{2}$-inverse strongly monotone, that is,

$$
\frac{\beta}{L^{2}}\|A x-A y\|^{2} \leq\langle x-y, A x-A y\rangle
$$

for all $x, y$ in the domain of $A$, and that $A^{-1}$ is also injective and $\beta$-inverse strongly monotone; see Section 2 for more details. Therefore, to find solutions to the variational inequality problem, we only need assume that $A$ is injective and inverse strongly monotone. Our results are based on the following theorem, which is known as Edelstein's theorem [7].

Theorem 1.5 ([7]) Let $C$ be a compact subset of a Hilbert space $H$ and $S$ be a contractive mapping of $C$ into $H$, that is,

$$
\|S x-S y\|<\|x-y\|
$$

for $x, y \in C$ with $x \neq y$. Furthermore, let $\left\{x_{n}\right\}$ be a sequence in $C$ defined by $u_{1} \in C$ and $x_{n+1}=S x_{n}$ for $n=1,2, \ldots$. Then $\left\{x_{n}\right\}$ converges strongly to a unique fixed point $v \in C$ of $S$.

\section{Preliminaries}

We denote the set of real numbers by $\mathbb{R}$ and the set of positive integers by $\mathbb{N}$. Furthermore, we denote a real Hilbert space by $H$, its inner product by $\langle\cdot, \cdot\rangle$, and the norm derived from the inner product by $\|\cdot\|$. Let $C$ be a subset of a Hilbert space $H$. We denote the identity mapping on $H$ by $I$. Let $T$ be a mapping of $C$ into $H$. Then $T$ is said to be

(i) firmly nonexpansive if for any $x, y \in C$,

$$
\|T x-T y\|^{2} \leq\langle x-y, T x-T y\rangle
$$

(ii) nonexpansive if for any $x, y \in C$,

$$
\|T x-T y\| \leq\|x-y\|
$$

(iii) $L$-Lipschitz continuous if there exists $L \in(1, \infty)$ such that for any $x, y \in C$,

$$
\|T x-T y\| \leq L\|x-y\|
$$

(iv) strictly contractive if there exists $r \in[0,1)$ such that for any $x, y \in C$,

$$
\|T x-T y\| \leq r\|x-y\|
$$

(v) contractive if for any $x, y \in C$ with $x \neq y$,

$$
\|T x-T y\|<\|x-y\|
$$

Obviously, a firmly nonexpansive mapping is also nonexpansive, and strict contractivity implies contractivity. We denote by $R(T)$ the range of $T$ and by $\operatorname{Fix}(T)$ the set of fixed points of $T$, that is, $\operatorname{Fix}(T)=\{x \in C: T x=x\}$. Fix $(T)$ is closed and convex when $T$ is nonexpansive [8-10]. In the Hilbert space setting, there is a fixed point existence result for nonexpansive mappings, which was proved by Browder [11, 12], Göhde [13] and Kirk [14]. 
Theorem 2.1 ([11-14]) Let $C$ be a bounded closed convex subset of a Hilbert space $H$ and $S$ be a nonexpansive self-mapping on $C$. Then $\operatorname{Fix}(S) \neq \emptyset$.

Let $C$ be a closed and convex subset of $H$. Then, for every point $x \in H$, there exists a unique nearest point in $C$, denoted by $P_{C}(x)$, such that $\left\|x-P_{C}(x)\right\| \leq\|x-y\|$ for all $y \in C$. $P_{C}$ is called the metric projection of $H$ onto $C$. We know that $P_{C}$ is a firmly nonexpansive mapping of $H$ onto $C$.

Let $a \in(0, \infty)$. Let $A$ be an operator sending $C$ into $H$. Then $A$ is said to be

(i) monotone if for any $x, y \in C$,

$$
\langle x-y, A x-A y\rangle \geq 0
$$

(ii) $\beta$-strongly monotone if there exists $\beta \in(0,1)$ such that for any $x, y \in C$,

$$
\beta\|x-y\|^{2} \leq\langle x-y, A x-A y\rangle
$$

(iii) $\beta$-inverse strongly monotone if there exists $\beta \in(0,1)$ such that for any $x, y \in C$,

$$
\beta\|A x-A y\|^{2} \leq\langle x-y, A x-A y\rangle .
$$

We know that if $A$ is $\beta$-inverse strongly monotone, for any $a \in(0,2 \beta), I-a A$ is a nonexpansive mapping of $C$ into $H$.

Let $A$ be an injective mapping of $C$ into $H$. Then there is an inverse mapping $A^{-1}$ of $A$ such that, for $x \in C$ and $y \in R(A), A^{-1} y=x$ if $A x=y$. It is obvious that $A^{-1}$ is also injective. Let $A$ be $\beta$-strongly monotone of $C$ into $H$. Then $A$ is injective. To see this, let us assume that $A x=A y$ for $x, y \in C$ with $x \neq y$. Then, from the strong monotonicity of $A$, we have

$$
\beta\|x-y\|^{2} \leq\langle x-y, A x-A y\rangle=0,
$$

and hence $x=y$. This is a contradiction. So, $A x \neq A y$. This means that $A$ is injective. If $A$ is also $L$-Lipschitz continuous, $A$ is $\beta / L^{2}$-inverse strongly monotone because

$$
\frac{\beta}{L^{2}}\|A x-A y\|^{2} \leq \frac{\beta}{L^{2}} L^{2}\|x-y\|^{2}=\beta\|x-y\|^{2} \leq\langle x-y, A x-A y\rangle .
$$

Many methods for solving the variational inequality problem are based on the following (see $[8,10]$ for instance).

Lemma $2.1([8,10])$ Let $C$ be a closed and convex subset of a Hilbert space $H$ and $A$ be a mapping of $C$ into $H$ and $a \in(0, \infty)$. Then $\operatorname{Fix}\left(P_{C}(I-a A)\right)=V I(C, A)$.

The following lemmas are presented without proof. The first lemma is known as Browder's demiclosedness principle $[11,15]$ (see also $[8,10]$ ).

Lemma 2.2 Let $C$ be a closed and convex subset of a Hilbert space $H$ and $S$ be a nonexpansive mapping of $C$ into itself. Let $\left\{x_{n}\right\}$ be a sequence in $C$ which converges weakly to $u \in C$ and satisfies $\lim _{n}\left\|S x_{n}-x_{n}\right\|=0$. Then $u \in \operatorname{Fix}(S)$.

The next lemma is also well known. 
Lemma 2.3 ([10]) Let $\left\{x_{n}\right\}$ be a sequence in a Hilbert space H. Assume that there is a point $z \in H$ such that any subsequence $\left\{x_{n_{j}}\right\}$ of $\left\{x_{n}\right\}$ has a subsequence which converges strongly (resp. weakly) to $z$. Then $\left\{x_{n}\right\}$ itself converges strongly (resp. weakly) to $z$.

\section{Injective $\boldsymbol{\beta}$-inverse strongly monotone operators}

As preparation for the next section, here we study the properties of injective $\beta$-inverse strongly monotone operators.

Lemma 3.1 Let $C$ be a subset of a Hilbert space $H$ and $A$ be a $\beta$-inverse strongly monotone operator sending $C$ into $H$. Then, for $a \in(0,2 \beta)$,

$$
\left\|A_{a} x-A_{a} y\right\|^{2} \leq\|x-y\|^{2}-a(2 \beta-a)\|A x-A y\|^{2}
$$

for $x, y \in C$, where $A_{a}=I-a A$. If $A$ is also injective, then $A_{a}$ is contractive.

Proof We have that for any $x, y \in C$,

$$
\begin{aligned}
\left\|A_{a} x-A_{a} y\right\|^{2} & =\|(I-a A) x-(I-a A) y\|^{2} \\
& =\|(x-y)-a(A x-A y)\|^{2} \\
& =\|x-y\|^{2}+a^{2}\|A x-A y\|^{2}-2 a\langle x-y, A x-A y\rangle \\
& \leq\|x-y\|^{2}+a^{2}\|A x-A y\|^{2}-2 a \beta\|A x-A y\|^{2} \\
& =\|x-y\|^{2}-a(2 \beta-a)\|A x-A y\|^{2} .
\end{aligned}
$$

When $A$ is injective, we have from $a \in(0,2 \beta)$ that for $x, y \in C$ with $x \neq y$,

$$
\left\|A_{a} x-A_{a} y\right\|^{2} \leq\|x-y\|^{2}-a(2 \beta-a)\|A x-A y\|^{2}<\|x-y\|^{2},
$$

and hence $A_{a}$ is contractive.

Corollary 3.1 Let $C$ be a subset of a Hilbert space H. Furthermore, $S$ be a nonexpansive mapping of $H$ into $C$ and $A$ be a $\beta$-inverse strongly monotone operator sending $R(S)$ into $H$. Then, for $a \in(0,2 \beta)$,

$$
\left\|U_{a} x-U_{a} y\right\|^{2} \leq\|x-y\|^{2}-a(2 \beta-a)\|A S x-A S y\|^{2}
$$

for $x, y \in H$, where $U_{a}=(I-a A) S$. If $A$ is also injective, then $U_{a}$ is contractive.

Proof Putting $A_{a}=I-a A$, we have from Lemma 3.1 and the nonexpansivity of $S$ that for any $x, y \in H$,

$$
\begin{aligned}
\left\|U_{a} x-U_{a} y\right\|^{2} & =\left\|A_{a} S x-A_{a} S y\right\|^{2} \\
& \leq\|S x-S y\|^{2}-a(2 \beta-a)\|A S x-A S y\|^{2} \\
& \leq\|x-y\|^{2}-a(2 \beta-a)\|A S x-A S y\|^{2} .
\end{aligned}
$$

If $A$ is injective, it is sufficient to check for $x, y \in H$ with $x \neq y$, as follows: 
(a) If $S x=S y$, it follows that $\left\|U_{a} x-U_{a} y\right\|=0$. So, $\left\|U_{a} x-U_{a} y\right\|<\|x-y\|$ for $x, y \in H$ with $x \neq y$.

(b) If $S x \neq S y, A S x \neq A S y$. So, it follows from (2) and $a \in(0,2 \beta)$ that

$$
\left\|U_{a} x-U_{a} y\right\|^{2} \leq\|x-y\|^{2}-a(2 \beta-a)\|A S x-A S y\|<\|x-y\|^{2}
$$

Lemma 3.2 Let $C$ be a closed convex subset of a Hilbert space H. Furthermore, let $A$ be an injective $\beta$-inverse strongly monotone operator sending $C$ into $H$ and $P_{C}$ be the metric projection of $H$ onto $C$. Then, for $a \in(0,2 \beta), P_{C}(I-a A)$ is contractive on $C$.

Proof Putting $A_{a}=I-a A$, we have from Lemma 3.1 and the nonexpansivity of $P_{C}$ that for $x, y \in C$ with $x \neq y$,

$$
\left\|P_{C} A_{a} x-P_{C} A_{a} y\right\| \leq\left\|A_{a} x-A_{a} y\right\|<\|x-y\| .
$$

Thus, $P_{C}(I-a A)$ is contractive.

Lemma 3.3 Let $C$ be a closed convex subset of a Hilbert space $H$ and $A$ be an injective $\beta$-inverse strongly monotone operator sending $C$ into $H$ with $\operatorname{VI}(C, A) \neq \emptyset$. Then $\operatorname{VI}(C, A)$ is a singleton.

Proof Assume that there exist $x, y \in V I(C, A)$ with $x \neq y$. Let $a \in(0,2 \beta)$. Then it follows from Lemma 2.1 that $x, y \in \operatorname{Fix}\left(P_{C} A_{a}\right)$, where $A_{a}=I-a A$. Moreover, since $A$ is injective, we can easily verify from Lemma 3.2 that

$$
\|x-y\|=\left\|P_{C} A_{a} x-P_{C} A_{a} y\right\|<\|x-y\| .
$$

This is a contradiction. Accordingly, we have the desired result.

Finally, we give an example of an injective inverse strongly monotone operator in $\mathbb{R}$ which is not strongly monotone.

Example 1 Let $\left\{a_{n}\right\} \subset(1,2]$ be a monotone decreasing sequence such that $a_{1}=2$ and $a_{n} \rightarrow 1$, and let $\left\{b_{n}\right\} \subset[0,1)$ be a monotone increasing sequence such that $b_{1}=0$ and $b_{n} \rightarrow 1$. Put $r_{n}:=a_{n}-a_{n+1}$ and $s_{n}:=b_{n+1}-b_{n}$ for each $n \in \mathbb{N}$. Define a function $f:[0,2] \rightarrow \mathbb{R}$ by

$$
f(x)= \begin{cases}-\frac{1}{s_{n}}\left(x-b_{n+1}\right)^{2}+\left(b_{n+1}-1\right) & \left(x \in\left[b_{n}, b_{n+1}\right]\right), \\ 0 & (x=1), \\ \frac{1}{r_{n}}\left(x-a_{n+1}\right)^{2}+\left(a_{n+1}-1\right) & \left(x \in\left[a_{n+1}, a_{n}\right]\right) .\end{cases}
$$

Obviously, $f$ is continuous and inverse strongly monotone, but not differentiable at $a_{n}$ and $b_{n}$ for all $n \geq 1$. Furthermore, $f^{-1}$ is not Lipschitz continuous; that is, $f$ is not strongly monotone.

In the above example, it is difficult to apply Newton's method and (HSDM), which are valid for continuous and differentiable mappings, or strongly monotone and Lipschitz 
continuous operators. Hence, there will be many injective inverse strongly monotone operators which are not strongly monotone. Our main results in the next section are effective for such operators.

\section{Main results}

In this section, we present iterative algorithms for solving the variational inequality problem for an injective inverse strongly monotone operator and their convergence analyses.

Theorem 4.1 Let $C$ be a closed convex subset of a Hilbert space $H$ and $A$ be an injective $\beta$-inverse strongly monotone operator sending $C$ into $H$ with $V I(C, A) \neq \emptyset$. Let $a \in(0,2 \beta)$. Let $\left\{u_{n}\right\}$ be a sequence generated by $u_{1} \in C$ and

$$
u_{n+1}=P_{C}(I-a A) u_{n}
$$

for all $n \in \mathbb{N}$. Then $\left\{A u_{n}\right\}$ converges strongly to $x_{0} \in H$ such that $A^{-1} x_{0}$ is the unique point in $\operatorname{VI}(C, A)$.

Proof From Lemma 3.3, $V I(C, A)$ is a singleton. Let $\{v\}=V I(C, A), x_{0}=A v$ and $A_{a}=I-a A$ for $a \in(0,2 \beta)$. Then, from Lemma 2.1, we know that $\{v\}=V I(C, A)=\operatorname{Fix}\left(P_{C} A_{a}\right)$. Moreover, from Lemma 3.1, we can see that, for any $n \in \mathbb{N}$,

$$
\begin{aligned}
\left\|u_{n+1}-v\right\|^{2} & =\left\|P_{C} A_{a} u_{n}-P_{C} A_{a} v\right\|^{2} \leq\left\|A_{a} u_{n}-A_{a} v\right\|^{2} \\
& \leq\left\|u_{n}-v\right\|^{2}-a(2 \beta-a)\left\|A u_{n}-A v\right\|^{2} \\
& =\left\|u_{n}-v\right\|^{2}-a(2 \beta-a)\left\|A u_{n}-x_{0}\right\|^{2} .
\end{aligned}
$$

Then $\left\{\left\|u_{n}-v\right\|^{2}\right\}$ is nonincreasing, and hence $\left\{\left\|u_{n}-v\right\|^{2}\right\}$ has a limit. Furthermore, it follows that for any $n \in \mathbb{N}$,

$$
a(2 \beta-a)\left\|A u_{n}-x_{0}\right\|^{2} \leq\left\|u_{n}-v\right\|^{2}-\left\|u_{n+1}-v\right\|^{2} .
$$

From $a(2 \beta-a)>0$, we get $\lim _{n \rightarrow \infty}\left\|A u_{n}-x_{0}\right\|=0$.

The following are direct consequences of Theorem 4.1.

Corollary 4.1 Let $C$ be a bounded closed and convex subset of a Hilbert space $H$ and $A$ be an injective $\beta$-inverse strongly monotone operator sending $C$ into $H$. Let $a \in(0,2 \beta)$. Let $\left\{u_{n}\right\}$ be a sequence generated by $u_{1} \in C$ and

$$
u_{n+1}=P_{C}(I-a A) u_{n}
$$

for all $n \in \mathbb{N}$. Then $\left\{A u_{n}\right\}$ converges strongly to $x_{0} \in H$ such that $A^{-1} x_{0}$ is the unique point in $\operatorname{VI}(C, A)$.

Proof Since $P_{C}(I-a A)$ is nonexpansive for $a \in(0,2 \beta)$, it follows from Theorem 2.1 and Lemma 2.1 that $\emptyset \neq \operatorname{Fix}\left(P_{C}(I-a A)\right)=V I(C, A)$. From Theorem 4.1, we reach the conclusion. 
Corollary 4.2 Let $C$ be a closed convex subset of a Hilbert space $H$ and $A$ be an injective $\beta$-inverse strongly monotone operator sending $C$ into $H$ with $V I(C, A) \neq \emptyset$ whose inverse is continuous on $R(A)$. Let $a \in(0,2 \beta)$. Let $\left\{u_{n}\right\}$ be a sequence generated by $u_{1} \in C$ and

$$
u_{n+1}=P_{C}(I-a A) u_{n}
$$

for all $n \in \mathbb{N}$. Then $\left\{u_{n}\right\}$ converges strongly to the unique point in $\operatorname{VI}(C, A)$.

Proof From Theorem 4.1, $\left\{A u_{n}\right\}$ converges strongly to $x_{0}$, where $A^{-1} x_{0}$ is the unique point in $\operatorname{VI}(C, A)$. Then it follows from the continuity of $A^{-1}$ that

$$
\lim _{n \rightarrow \infty}\left\|u_{n}-A^{-1} x_{0}\right\|=\lim _{n \rightarrow \infty}\left\|A^{-1} A u_{n}-A^{-1} x_{0}\right\|=0 .
$$

This completes the proof.

The following theorem is derived directly from Theorem 1.5 and Lemma 3.1.

Theorem 4.2 Let $C$ be a compact convex subset of a Hilbert space $H$ and $A$ be an injective $\beta$-inverse strongly monotone operator sending $C$ into $H$. Let $a \in(0,2 \beta)$. Let $\left\{u_{n}\right\}$ be a sequence in $C$ generated by $u_{1} \in C$ and

$$
u_{n+1}=P_{C}(I-a A) u_{n}
$$

for all $n \in \mathbb{N}$. Then $\left\{u_{n}\right\}$ converges strongly to the unique point in $\operatorname{VI}(C, A)$.

Proof From Lemma 3.1, $P_{C}(I-a A)$ is a contractive self-mapping on $C$. Then, by Theorem $1.5,\left\{u_{n}\right\}$ converges strongly to the unique fixed point $v$ in $\operatorname{Fix}\left(P_{C}(I-a A)\right)$. From Lemma 2.1, we know $\operatorname{Fix}\left(P_{C}(I-a A)\right)=V I(C, A)$. Thus, $v$ is the unique point in $V I(C, A)$.

Finally, the following theorem due to Yamada [4] is connected with Theorem 1.2.

Theorem 4.3 Let $C$ be a bounded subset of a Hilbert space $H$ and $S$ be a nonexpansive mapping of $H$ into $C$. Let $A$ be an injective operator sending $C$ into $H$ and $\beta$-inverse strongly monotone on $R(S)$. Let $\left\{c_{n}\right\}$ be a sequence in $(0,2 \beta)$ with $\lim _{n \rightarrow \infty} c_{n}=0$. Let $\left\{u_{n}\right\}$ be a sequence in $H$ generated by

$$
u_{n}=\left(I-c_{n} A\right) S u_{n}
$$

for all $n \in \mathbb{N}$. Then $\left\{A S u_{n}\right\}$ converges strongly to $x_{0} \in H$ such that $A^{-1} x_{0}$ is the unique point in $\operatorname{VI}(\operatorname{Fix}(S), A)$.

Proof Put $U_{c_{n}}:=\left(I-c_{n} A\right) S$ for all $n \in \mathbb{N}$. From the boundedness of $C$ and the Lipschitz continuity of $A, R(A)$ is bounded. Then $\bigcup_{a \in[0,2]} a R(A)$ and $C-\bigcup_{a \in[0,2]} a R(A)$ are also bounded. So, there exists $r>0$ such that

$$
\operatorname{Fix}(S) \subset R(S) \subset C \subset C-\bigcup_{a \in[0,2]} a R(A) \subset B_{r}
$$


where $B_{r}$ is a closed ball with center 0 and radius $r$. Accordingly, we can consider $S$ and each $U_{c_{n}}$ to be self-mappings on $B_{r}$. From Theorem 2.1, it follows that Fix $(S)$ is nonempty, bounded, closed and convex. From Lemma 3.3, we also know that $V I(\operatorname{Fix}(S), A)$ is a singleton in $\operatorname{Fix}(S)$. From Corollary 3.1, each $U_{c_{n}}$ is a contractive self-mapping on $B_{r}$; that is, each $U_{c_{n}}$ has a unique fixed point in $B_{r}$. Then we can take a sequence $\left\{u_{n}\right\} \subset B_{r}$ such that $U_{c_{n}} u_{n}=u_{n}$. From the boundedness of $\left\{u_{n}\right\},\left\{A S u_{n}\right\}$ is also bounded. Let $\left\{A S u_{n_{j}}\right\}$ be any subsequence of $\left\{A S u_{n}\right\}$. Then, from the boundedness of $\left\{u_{n_{j}}\right\}$, there exists a weakly convergent subsequence of $\left\{u_{n_{j}}\right\}$. By passing to subsequences, we may assume that $\left\{u_{n_{j}}\right\}$ itself converges weakly to some $u \in B_{r}$. We have that for any $n \in \mathbb{N}$,

$$
\left\|S u_{n}-u_{n}\right\|=\left\|S u_{n}-U_{c_{n}} u_{n}\right\|=\left\|S u_{n}-\left(I-c_{n} A\right) S u_{n}\right\|=c_{n}\left\|A S u_{n}\right\| .
$$

Since $\left\{A S u_{n}\right\}$ is bounded and $\lim _{n \rightarrow \infty} c_{n}=0$, we find that

$$
\lim _{n \rightarrow \infty}\left\|S u_{n}-u_{n}\right\|=0 .
$$

From the demiclosedness of $S$, we get $u \in \operatorname{Fix}(S)$. Let $\{v\}=V I(\operatorname{Fix}(S), A)$ and $x_{0}=A v$. Since $v \in \operatorname{Fix}(S)$, we also know that for any $n \in \mathbb{N}$,

$$
U_{c_{n}} v-v=\left(I-c_{n} A\right) S v-v=\left(I-c_{n} A\right) v-v=-c_{n} A v .
$$

Corollary 3.1 implies that for any $j \in \mathbb{N}$,

$$
\begin{aligned}
c_{n_{j}}\left(2 \beta-c_{n_{j}}\right)\left\|A S u_{n_{j}}-A v\right\|^{2} & =c_{n_{j}}\left(2 \beta-c_{n_{j}}\right)\left\|A S u_{n_{j}}-A S v\right\|^{2} \\
& \leq\left\|u_{n_{j}}-v\right\|^{2}-\left\|U_{c_{n_{j}}} u_{n_{j}}-U_{c_{n_{j}}} v\right\|^{2} \\
& =\left\|u_{n_{j}}-v\right\|^{2}-\left\|u_{n_{j}}-\left(I-c_{n_{j}} A\right) S v\right\|^{2} \\
& =\left\|u_{n_{j}}-v\right\|^{2}-\left\|u_{n_{j}}-\left(I-c_{n_{j}} A\right) v\right\|^{2} \\
& =\left\|u_{n_{j}}-v\right\|^{2}-\left\langle u_{n_{j}}-v+c_{n_{j}} A v, u_{n_{j}}-v+c_{n_{j}} A v\right\rangle \\
& =\left\|u_{n_{j}}-v\right\|^{2}-\left\|u_{n_{j}}-v\right\|^{2}-2\left\langle u_{n_{j}}-v, c_{n_{j}} A v\right\rangle-c_{n_{j}}^{2}\|A v\|^{2} \\
& =-2 c_{n_{j}}\left\langle u_{n_{j}}-v, A v\right\rangle-c_{n_{j}}^{2}\|A v\|^{2} .
\end{aligned}
$$

From $c_{n_{j}}>0$, we get

$$
\frac{2 \beta-c_{n_{j}}}{2}\left\|A S u_{n_{j}}-A v\right\|^{2}+\frac{c_{n_{j}}}{2}\|A v\|^{2} \leq-\left\langle u_{n_{j}}-v, A v\right\rangle .
$$

Since $\left\{u_{n_{j}}\right\}$ converges weakly to $u \in \operatorname{Fix}(S), v \in V I(\operatorname{Fix}(S), A), \lim _{j \rightarrow \infty} c_{n_{j}}=0$ and the boundedness of $\left\|A S u_{n_{j}}-A v\right\|^{2}$, we also get

$$
\beta \limsup _{j \rightarrow \infty}\left\|A S u_{n_{j}}-A v\right\|^{2} \leq-\langle u-v, A v\rangle \leq 0 .
$$

So, we have that $\left\{A S u_{n_{j}}\right\}$ converges strongly to $A v$. This means that any subsequence of $\left\{A S u_{n}\right\}$ has a subsequence which converges strongly to $A v$. Thus, from Lemma 2.3, $\left\{A S u_{n}\right\}$ itself converges strongly to $A v=x_{0}$, where $v$ is the unique point in $\operatorname{VI}(\operatorname{Fix}(S), A)$. 
Corollary 4.3 Let $C$ be a bounded subset of a Hilbert space $H$ and $S$ be a nonexpansive mapping of $H$ into $C$. Let $A$ be an injective operator sending $C$ into $H$ and $\beta$-inverse strongly monotone on $R(S)$ whose inverse is continuous on $R(A)$. Let $\left\{c_{n}\right\}$ be a sequence in $(0,2 \beta)$ with $\lim _{n \rightarrow \infty} c_{n}=0$. Let $\left\{u_{n}\right\}$ be a sequence in $H$ generated by

$$
u_{n}=\left(I-c_{n} A\right) S u_{n}
$$

for all $n \in \mathbb{N}$. Then $\left\{u_{n}\right\}$ converges strongly to the unique point in $\operatorname{VI}(\operatorname{Fix}(S), A)$.

Proof By Theorem 4.3, $\left\{A S u_{n}\right\}$ converges strongly to $x_{0}$, where $A^{-1} x_{0}=: v$ is the unique solution of $\operatorname{VI}(\operatorname{Fix}(S), A)$. From the continuity of $A^{-1}$, we have

$$
\lim _{n \rightarrow \infty}\left\|S u_{n}-v\right\|=\lim _{n \rightarrow \infty}\left\|A^{-1} A S u_{n}-A^{-1} x_{0}\right\|=0 .
$$

Accordingly, we have that for all $n \in \mathbb{N}$,

$$
\left\|u_{n}-v\right\| \leq\left\|u_{n}-S u_{n}\right\|+\left\|S u_{n}-v\right\|
$$

Thus, from (3), we find that $\lim _{n \rightarrow \infty}\left\|u_{n}-v\right\|=0$, and we have reached the conclusion.

Remark 4.1 The conditions of $A$ in Theorem 4.3 and Corollary 4.3 are weaker than that of $A$ in Theorem 3.2 of [4]. However, we assumed that $C$ is bounded.

\section{Competing interests}

The author declares that he has no competing interests.

\section{Acknowledgements}

The author would like to thank the referees for their valuable suggestions on the paper.

Received: 14 February 2015 Accepted: 22 May 2015 Published online: 13 June 2015

\section{References}

1. Lions, JL, Stampacchia, G: Variational inequalities. Commun. Pure Appl. Math. 20, $493-519$ (1967)

2. Goldstein, AA: Convex programming in Hilbert space. Bull. Am. Math. Soc. 70, 709-710 (1964)

3. Zeidler, E: Nonlinear Functional Analysis and Its Applications. III: Variational Methods and Optimization. Springer, Berlin (1985); Translated from the German by Leo F. Boron

4. Yamada, I: The hybrid steepest descent method for the variational inequality problem over the intersection of fixed point sets of nonexpansive mappings. In: Butnariu, D, Censor, Y, Reich, S (eds.) Inherently Parallel Algorithms in Feasibility and Optimization and Their Applications, pp. 473-504. North-Holland, Amsterdam (2001)

5. Xu, HK, Kim, TH: Convergence of hybrid steepest-descent methods for variational inequalities. J. Optim. Theory Appl. 119, 185-201 (2003)

6. Banach, S: Sur les opérations dans les ensembles abstraits et leurs applications. Fundam. Math. 3, 133-181 (1922)

7. Edelstein, M: On fixed and periodic points under contractive mappings. J. Lond. Math. Soc. 37, 74-79 (1962)

8. Bauschke, HH, Combettes, PL: Convex Analysis and Monotone Operator Theory in Hilbert Spaces. CMS Books in Mathematics. Ouvrages de Mathématiques de la SMC. Springer, Berlin (2011); With a foreword by Hédy Attouch

9. Goebel, K, Kirk, WA: Topics in Metric Fixed Point Theory. Cambridge University Press, Cambridge (1990)

10. Takahashi, W: Nonlinear Functional Analysis. Fixed Point Theory and Its Applications. Yokohama Publishers, Yokohama (2000)

11. Browder, FE: Fixed-point theorems for noncompact mappings in Hilbert space. Proc. Natl. Acad. Sci. USA 53, $1272-1276(1965)$

12. Browder, FE: Nonexpansive nonlinear operators in a Banach space. Proc. Natl. Acad. Sci. USA 54, 1041-1044 (1965)

13. Göhde, D: Zum Prinzip der kontraktiven Abbildung. Math. Nachr. 30, 251-258 (1965)

14. Kirk, WA: A fixed point theorem for mappings which do not increase distances. Am. Math. Mon. 72, 1004-1006 (1965)

15. Opial, Z: Weak convergence of the sequence of successive approximations for nonexpansive mappings. Bull. Am. Math. Soc. 73, 591-597 (1967) 